\title{
TESTABILITY WITH UNBOUNDED TESTING STRATEGIES
}

\author{
Bernd Baumgarten, Olaf Henniger \\ GMD - German National Research Center for Information Technology \\ Rheinstr. 75, 64295 Darmstadt, Germany \\ \{baumgart,henniger\}@darmstadt.gmd.de
}

\begin{abstract}
Testability and design for testability are widely discussed practical issues in software engineering, especially in protocol engineering. A number of basic testability qualities were defined formally and independently from any special system model. In this paper we refine these notions on the one hand by a containment order on experiments and on the other hand by a formal distinction between bounded and unbounded experimentation. Containment and bounds will be interpreted mainly temporally, but can also more generally be considered as referring to a "width of view", of which time is only one, though prominent, aspect.
\end{abstract}

Keywords: Semantic foundations, testing, testability, specification theory

\section{INTRODUCTION}

In theory and practice of testing, testability is often emphasized as a desirable property of specifications and systems. There exist various useful informal discussions of testability $[5,7,8,9,10]$. In [3], a general semantic framework was used to discuss general qualitative concepts of testability, formalizing what it means to demand that conformance or non-conformance to a specification can be decided by testing. In the present paper, we expand the approach of [3] and explore qualitative testability properties that make sense if unbounded strategies of finite experiments are available. These properties are of interest either if the nature of the tested systems guarantees termination of the strategies, or if it is so important at least to attempt the strategies that the risk that they do not terminate and have to be aborted is accepted. 
This paper is part of an effort to introduce a general theory of specification as a basic common language for specification, verification, and testing [4]. It is organized as follows: Section 2 gives a general definition for specification contexts. Section 3 reviews weak/strong non-deterministic/deterministic refutability/validatability as qualitative notions of testability, which were discussed in detail in [3]. Section 4 distinguishes between bounded and unbounded experimentation. Section 5 defines refutability and validatability by strategies and compares them with the corresponding properties defined w.r.t. single experiments. Section 6 gives conclusions and an outlook.

\section{SPECIFICATION CONTEXTS}

\subsection{DEFINITION}

When dealing with specifications, we usually do so within some context, characterized by a population of systems under consideration, the set of their possibly relevant properties, and a set of possible observations of interest.

In [2], a specification context is defined as a quintuple Cont $=($ Systs, Props, Obs, has_property, permits) where Systs is a set of systems, Props is a set of properties, Obs is a set of observations, has_property is a relation between systems and properties, and permits is a relation between systems and observations.

Specification contexts $[2,4]$ form a simple and pervasive paradigm in which notions of specification and test can be formulated, independently of particular formal description techniques. A specification context shall fulfil the following non-degeneracy assumptions:

Systs $\neq \emptyset \wedge O b s \neq \emptyset$.

$\forall$ sys $\in$ Systs $: \exists o b s \in O b s:$ sys permits obs.

$\forall$ obs $\in$ Obs $: \exists$ sys $\in$ Systs $:$ sys permits obs.

These assumptions are based on practical considerations. A specification context without systems or observations is of little interest. Any relevant system will be observable by at least one observation, and any relevant observation will be possible on at least one system.

What is practically considered as a system, a property, or an observation in a context depends on the chosen part of reality or field of thinking, the chosen level of abstraction, and the envisaged meaningful uses and abuses of the system. In this paper we will not be concerned with Props and has_property. 


\subsection{SPECIFICATIONS AND CONFORMANCE}

The (visible) behaviour of a system with respect to a specification context Cont consists of all observations that can be made of the system:

sys_beh: $\begin{cases}\text { Systs } & \rightarrow \mathcal{P}(\text { Obs }) \\ \text { sys } & \mapsto\{\text { obs } \in \text { Obs } \mid \text { sys permits obs }\} .\end{cases}$

In general, any subset $B e h \subseteq O b s$ of observations defines a behaviour.

Loosely spoken, a specification is "anything that defines a behaviour," i.e. a set of permitted observations. This set is usually not listed literally, in particular if it is infinite. Rather, it is syntactically represented by a member of a set Specs of specification terms, by way of a pre-determined observational semantics obs_sem : Specs $\rightarrow \mathcal{P}(O b s)$, cf. Figure 1 .

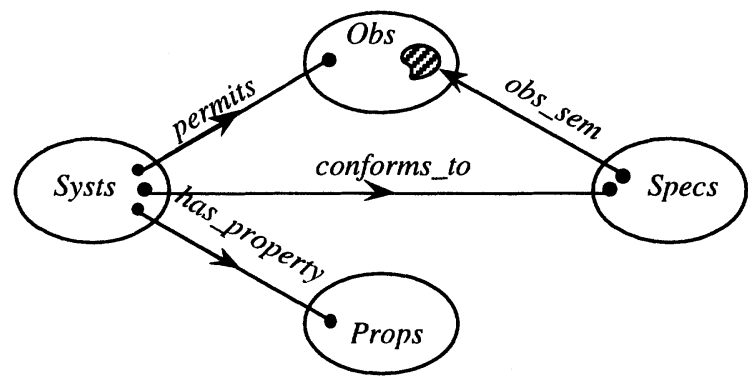

Figure 1 The semantic embedding of specifications into a specification context.

A specification spec is called contradictory if obs_sem (spec) $=\emptyset$, and void if $o b s_{-} s e m(s p e c)=O b s$, and non-degenerate if neither. Non-trivial aspects of voidness have been investigated in [2].

A system sys conforms to a specification (term) spec if its behaviour stays within the range allowed by spec:

sys conforms_to spec $: \Leftrightarrow$ sys_beh (sys) $\subseteq$ obs_sem (spec).

As temporary inactivity is observable in practical contexts, one can avoid the well-known disadvantage of untimed trace semantics that total inactivity is always conforming.

The above framework is fairly general and applies not only to digital information processing systems.

\subsection{EXPERIMENTAL SPECIFICATION CONTEXTS}

To distinguish between the (system-independent) experiments performed on a system and their possible (usually both experiment- and system-dependent) outcomes, the general definition of specification context is refined. 
In [3], an experimental specification context is defined as a specification context ExpCont $=($ Systs, Props, Obs, has_property, permits $)$, fulfilling the Assumptions i, ii, and iii, where Obs is the Cartesian product Exps $\times$ Outs of a set of experiments and a set of outcomes. Experimental specification contexts are related with the observation frameworks of [6] and even closer with the observation schemes of [1].

The possibility that the experiment exp performed on system sys yields the outcome out is represented by sys permits (exp,out), and is also written as (sys, exp) may_yield out.

poss_outs(sys, exp) $:=\{$ out $\in$ Outs $\mid($ sys, exp $)$ may_yield out $\}$

denotes the set of all possible outcomes of exp performed on a system sys. We assume that in an experimental specification context every experiment performed on a system yields at least one outcome,

$\forall$ sys $\in$ Systs, exp $\in$ Exps : poss_outs(sys, exp) $\neq \emptyset$,

such that may_yield is left-total. This means practically that we do not allow eternal experiments, and that we treat test log entries like "nothing happened, so we broke off after one hour" or "test equipment could not be connected to the system under test" as possible outcomes.

The definition of a specification carries over without much change, such that a specification defines which outcomes may be yielded by which experiments. We call an outcome out allowed for a specification spec and an experiment exp if $($ exp, out $) \in$ obs_sem (spec). Otherwise, we call it forbidden. Allowed outcomes form the set

allowed $($ spec, exp $):=\{$ out $\in$ Outs $\mid(\exp$, out $) \in$ obs_sem $($ spec $)\}$.

By our definitions, in an ExpCont, a system sys conforms to a specification spec if the system may yield only allowed outcomes:

sys conforms_to spec $\Leftrightarrow$ poss_outs (sys, exp) $\subseteq$ allowed (spec, exp).

We call an outcome out valid for a specification spec and an experiment exp if it may have come from a conforming system, i.e. if it is a member of the set

$$
\begin{aligned}
& \operatorname{valid}(\text { spec }, \text { exp }):=\{\text { out } \in \text { Outs } \mid \\
& \quad \exists \text { sys } \in \text { Systs }: \text { sys conforms_to spec } \wedge(\text { sys, exp }) \text { may_yield out }\} .
\end{aligned}
$$

Otherwise, we call it invalid. Invalid outcomes tell us that the investigated system is non-conforming. They form the set

invalid (spec, exp) $:=$ Outs $\backslash \operatorname{valid}($ spec, exp).

We call an outcome out validating for a specification spec and an experiment exp if it definitely comes from a conforming system, i.e. if it is a member of 
the set

$$
\begin{aligned}
& \text { validating }(\text { spec }, \text { exp }):=\{\text { out } \in \text { Outs } \mid \\
& \quad \forall \text { sys } \in \text { Systs }:(\text { sys }, \text { exp }) \text { may_yield out } \Rightarrow \text { sys conforms_to spec }\} .
\end{aligned}
$$

In practical contexts, this set will often be empty.

\section{FLAVOURS OF TESTABILITY}

\subsection{A TAXONOMY OF TESTABILITY}

The term "testability" can refer to specifications, systems, and testing environments. In this paper, we deal with qualitative aspects of the testability of a specification, asking whether, through testing, conformance to this specification can be

- refuted or validated

- for arbitrary non-conforming or conforming systems (strongly), only for some (weakly), or for none of them,

- possibly only with luck (non-deterministically, $N D$ ) or with a guarantee (deterministically, $D$ ),

- in bounded or arbitrary finite time.

The first three aspects have been discussed and illustrated by examples and/or counterexamples in [3]. In the present paper we recall the main results in order to refine them in view of the time aspect.

"Through testing" means that information about the behaviour of the system "under test" is procured only by means of observations. Observers do not know in advance the full behaviour of the system "under test", nor will they necessarily know after any combination of observations. For many contexts (in particular black-box contexts for reactive systems), the full behaviour of a system cannot be identified by means of observations, due to non-determinism and infinite cardinality. On the other hand, advance knowledge, or assumptions, about a system may save observation work and may even permit the determination of the full system behaviour.

\subsection{REFUTABILITY AND VALIDATABILITY IN EXPERIMENTAL SPECIFICATION CONTEXTS}

In ExpCont, a non-void specification spec is defined to be

- ND-refutable if any given non-conforming system can be shown to be non-conforming, though possibly only with luck, i.e. if 
$\forall$ sys $\in$ Systs $: \neg($ sys conforms_to spec $) \Rightarrow$ $\exists$ exp $\in$ Exps, out $\in$ invalid (spec, exp) : (sys, exp) may_yield out;

- weakly $D$-refutable if some non-conforming system can definitely be shown to be non-conforming, i.e. if

$\exists$ sys $\in$ Systs, exp $\in$ Exps $:$ poss_outs $($ sys, exp $) \subseteq$ invalid $($ spec, exp $)$;

- strongly $D$-refutable if any non-conforming system can definitely be shown to be non-conforming, i.e. if

$\exists$ exp $\in$ Exps $: \forall$ sys $\in$ Systs $: \neg($ sys conforms_to spec $) \Rightarrow$ poss_outs $($ sys, exp $) \subseteq$ invalid (spec, exp).

Fact 1 Every non-void specification spec is ND-refutable [3].

What is defined as ND-refutability might also be called strong ND-refutability as it allows to reveal any non-conforming system. Due to Fact 1, however, there is no point in differentiating between strong and weak ND-refutability.

In ExpCont, a non-contradictory specification spec is defined to be

- weakly ND-validatable if some conforming system can be shown to be conforming, though possibly only with luck, i.e. if

$\exists$ sys $\in$ Systs, exp $\in$ Exps, out $\in$ validating $($ spec, exp $):$

(sys, exp) may_yield out;

- strongly $N D$-validatable if any given conforming system can be shown to be conforming, though possibly only with luck, i.e. if

$\forall$ sys $\in$ Systs $:$ sys conforms_to spec $\Rightarrow$

$\exists \exp \in$ Exps, out $\in$ validating $($ spec, $\exp ):$

(sys, exp) may_yield out;

- weakly $D$-validatable if some conforming system can definitely be shown to be conforming, i.e. if

$\exists$ sys $\in$ Systs, exp $\in$ Exps $:$ poss_outs $($ sys, exp $) \subseteq$ validating $($ spec, exp $)$; 
- strongly $D$-validatable if any conforming system can definitely be shown to be conforming, i.e. if

$$
\begin{gathered}
\exists \text { exp } \in \text { Exps }: \forall s y s \in \text { Systs }: \text { sys conforms_to spec } \Rightarrow \\
\text { poss_outs }(\text { sys }, \text { exp }) \subseteq \text { validating }(\text { spec }, \text { exp }) .
\end{gathered}
$$

Practically, validatability occurs only in rather special contexts.

Fact 2 The implications indicated in Figure 2 hold for non-degenerate specifications [3].

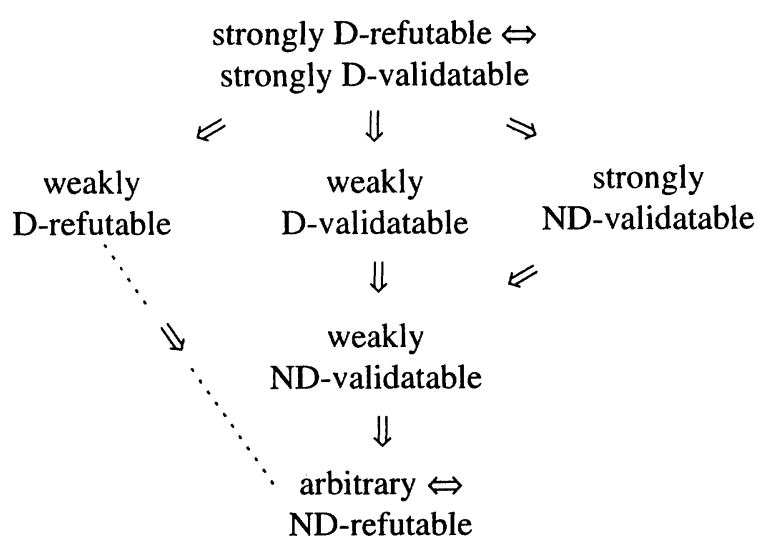

Figure 2 Implications between testability properties.

\section{BOUNDED AND UNBOUNDED EXPERIMENTATION \\ 4.1 OBSERVATIONS ARE FINITE}

Up to this point, we have not dealt with questions of duration or complexity of observations, experiments or outcomes.

Nobody has ever performed, or will ever perform, an observation that takes infinitely long. An observation or outcome only achieved after an infinite time span is not achieved in this world. Requirements referring directly or indirectly to infinitely long observations will often be practically void [2], and are then of little interest to all parties economically involved: customers or contractors, implementers, users or testers. Therefore, we consider each observation as obtained in a finite time interval. Similarly, in experimental specification contexts, we consider each outcome as obtained by the performance of an experiment in finite time. 


\subsection{WHAT ABOUT EXPERIMENTS?}

The definitions and assumptions introduced above, imply that each experiment performed on a system yields an outcome, and does so in finite time. A different question is whether experiments are bounded, i.e. whether for every experiment there is a pre-defined time limit within which it leads to an outcome. Of course, an experiment exp can be temporally unbounded, even though it yields for every system some outcome in finite time: let Systs contain infinitely many systems $s y s_{1}, s y s_{2}, \ldots$, and let it take $n$ seconds for the outcome to appear if exp is performed on system $s s_{n}$.

In commercial testing, usually only a previously fixed period of time is available for the performance of test cases (experiments) and test suites (sets or sequences of experiments). Unbounded testing is thus admittedly of lesser practical importance. It will ordinarily only be employed in high-risk systems. Then it pays to perform permanent testing, probably even in parallel with the operational phase, in order to uncover faults in a controllable way, before they would occur in an uncontrolled manner during regular operation.

\subsection{UNBOUNDED EXPERIMENTATION}

If we intuitively permitted experiments to be unbounded, then we could now proceed to partition Exps into bounded and unbounded experiments. Due to the practical predominance of bounded tests, we consider instead each experiment silently as bounded, i.e. as previously equipped with an upper bound of "effort" (duration and/or complexity). On this basis, we define in Section 4.5 something new to represent what we intuitively mean by unbounded experimentation, namely an "unbounded strategy." There will also be "bounded strategies," but they will not carry further than single experiments. We will see that unbounded testing can show more than bounded testing with some system populations.

Example A Let Systs $_{A}$ be the set of all finite binary sequences. We assume that the end of a sequence is recognizable: say, the sequences are read out at one figure per second, and a 2-second gap indicates termination. As one can stop listening to the sequence any time, the set of observations permitted by a system sys is identified with $\operatorname{Pref}($ sys), the set of all initial segments. Thus, $O b s_{A}=$ Systs $_{A}$. Consider, with the obvious observational semantics, the simple specification

$\operatorname{spec}_{A 1}$ : the system is a sequence of 0 's.

Theoretically, a guaranteed way both to refute and to validate the conformance of a system in finite time is to sit down and listen to the entire sequence. The end of a sequence of all 0 's validates conformance to $s p e c_{A 1}$, while the first figure 1 refutes it, both in finite time. In practice, however, one may die of 
old age (or a listening machine may fall apart due to wear) before a very long sequence is completely read out.

In fact, from a practical point of view, when one starts an activity, it does not really matter too much if this activity is guaranteed to terminate within some unknown finite time interval, or if the activity may not terminate at all. A finite duration may be too long for us to wait through it to detect finiteness, and an infinite duration can never be verified to be infinite. These questions are related with a problem of fairness investigated in [2].

How are we going to model unbounded experimentation without giving up our intuitive principle of bounded durations, not only of observations and outcomes, but even of experiments? One possible solution is to consider unbounded experimentation as an infinite sequence of experiments.

Example A (continued) In Example A, we can consider unbounded listening until the end of the sequence as a sequence of experiments $\left(\exp _{0}, \exp _{1}, \ldots\right)$ where each $\exp _{n}$ amounts to listening to the first $\min (n$, length $($ sys $))$ members of sys. This strategy can be broken off as soon as we know what we wanted to find out, i.e. after the first figure 1 or at the end of an all- 0 sequence.

Let us also consider an example where it is, informally spoken, impossible to find out everything about a system in finite time.

Example B Let Systs $_{B}$ be the set of all infinite binary sequences. We assume that the sequences are read out, on request, at one figure per second. As one can, and by necessity eventually will, stop listening to the sequence after a finite time interval, the set of observations permitted by a system sys is identified with Pref(sys), the set of all (finite) initial segments. Thus, Obs ${ }_{B}=$ Syst $_{A}$. Further below we will use, with the obvious observational semantics, three different simple specifications:

$\operatorname{spec}_{B 1}$ : the system is a sequence of 0 's.

$\operatorname{spec}_{B 2}$ : the system contains at least one figure 1.

$\operatorname{spec}_{B 3}$ : the system contains infinitely many figures 1 .

\subsection{AN ORDER ON EXPERIMENTS}

In Example A, we may consider all the experiments $\exp _{n}$ as started simultaneously, namely as soon as we sit down and listen to the sequence. As soon as we stop listening, say after the $n_{0}$-th sequence member, almost all of the experiments $\left(n>n_{0}\right)$ will be aborted.

Mark the obvious ordering between experiments of different "strength" (duration, complexity) which also determines an ordering between the outcomes with respect to the amount of information they give us. 
Mathematically, this approach prompts the following definitions. An ordered experimental specification context is a triple OrdExpCont $=($ ExpCont, contained_in, restr $)$ where

- ExpCont $=($ Systs, Props, Obs, has_property,permits $)$ is an experimental specification context with experiments in Exps and outcomes in Outs,

- contained_in is a partial order on Exps, and

- restr is a function, representing intuitively restriction to "smaller" experiments, that maps outcomes and experiments to outcomes such that

$$
\begin{gathered}
\exp _{1} \text { contained_in } \exp _{2} \Rightarrow \forall \text { sys } \in \text { Systs }: \text { poss_outs }\left(\text { sys }, \exp _{1}\right)= \\
\left\{\text { restr }\left(\text { out }_{2}, \exp _{1}\right) \in \text { Outs } \mid \text { out }_{2} \in \text { poss_outs }\left(\text { sys }, \exp _{2}\right)\right\} .
\end{gathered}
$$

We assume that restr is given effectively, such that what we can find out by an experiment would also be part of what can be found out by any "bigger" experiment, in which it is contained. Note that the application of restr is independent of the system observed.

As an illustration consider the scenario of the binary sequences in Example A, setting $\exp _{i}$ contained_in $\exp _{j}: \Leftrightarrow i \leq j$. Restriction to $\exp _{i}$ would then mean ignoring all sequence members after the $i$-th.

Let us consider two examples where contained_in is rather connected with "diagnostic breadth" than with duration.

Example C Assume each system sys $\in$ Systs $_{C}$ is a natural number, and that for each experiment $\exp _{n}$ the only outcome is

- -1 , if sys $>n$, and

- the number sys, if sys $\leq n$.

Example D Assume each sys $\in$ Systs $_{D}$ is a real number, and that every experiment $\exp _{n}$ consists in finding the interval $\left[x \cdot 2^{-n},(x+1) \cdot 2^{-n}\right), x$ an integer, in which sys is contained. We could imagine that the system can be handed over in an instant, e.g. by someone pointing, with a very fine pointer, to a position on a yardstick. In each of the above experiments, an analysis is made to find the interval in which the point is contained, on a grid of some chosen degree of fineness.

In the Examples $\mathrm{C}$ and $\mathrm{D}$, the temporal aspect is not immediately apparent; rather, the "breadth of view" or "level of detail" is what distinguishes the experiments from one another. However, from a practical point of view, temporal aspects can be identified. In Example C, it usually takes longer to read bigger numbers. In Example D, it takes longer to find out the membership to smaller intervals - think of how long it takes to procure more powerful microscopes. 


\subsection{STRATEGIES OF INCREASINGLY ORDERED EXPERIMENTS}

Turning now to "unbounded experiments" in OrdExpCont, we define a strategy as a growing sequence strat $=\left(\exp _{n}\right)_{n=1,2, \ldots}$ of experiments such that

$\forall m \geq 1: \exp _{m}$ contained_in $\exp _{m+1}$.

Let Strats be the set of all strategies.

We call a strategy strat bounded if its members are bounded above by some fixed experiment, i.e.

$\exists \exp \in$ Exps $: \forall n \geq 1: \exp _{n}$ contained_in exp;

otherwise strat is unbounded. With the aid of a bounded strategy, a tester cannot find out more about an unknown system than by the single experiment exp dominating the strategy. An unbounded strategy, however, may tell more about a system than any single experiment.

Intuitively, in all our examples, we will find out more and more about the system, the longer we follow a strategy. The examples are, however, distinct in one respect. In Examples $\mathrm{A}$ and $\mathrm{C}$, with the obvious strategy ( $\exp _{n}$ checks through the first $n$ sequence members, as far as they exist), we are guaranteed to know everything about the system in a finite time, even though - with the investigated system being unknown - we will not know in advance when this will be the case. No single experiment allows this in the two examples. In Examples B and D, no strategy allows us to identify the system completely.

5. TESTABILITY BY STRATEGIES

\subsection{TRUE EXTENSIONS OF THE TAXONOMY OF TESTABILITY}

The notions of testability introduced so far (Section 3.2) refer to "experimental" testability, i.e. - according to the boundedness assumption at the beginning of Section 4.3 - testability by experiments within a bounded finite time. Now we try to capture formally the additional power gained by using strategies.

In OrdExpCont, a non-void specification spec is defined to be strategically strongly $D$-refutable if, with an appropriate sequence of experiments, any given non-conforming system could definitely be shown to be non-conforming, i.e. if

$$
\begin{gathered}
\exists\left(\text { exp }_{n}\right)_{n=1,2, \ldots} \in \text { Strats }: \forall \text { sys } \in \text { Systs }: \neg(\text { sys conforms_to spec }) \Rightarrow \\
\exists m \in \mathbb{N}: \text { poss_outs }\left(\text { sys }, \text { exp }_{m}\right) \subseteq \text { invalid }\left(\text { spec }, \text { exp }_{m}\right) .
\end{gathered}
$$

In Example A, $\operatorname{spec}_{A 1}$ is strategically strongly D-refutable but not strongly D-refutable. 
In OrdExpCont, a non-contradictory specification spec is defined to be strategically strongly $D$-validatable if, with an appropriate sequence of experiments, any given conforming system could definitely be shown to be conforming, i.e. if

$$
\begin{gathered}
\exists\left(\exp _{n}\right)_{n=1,2, \ldots} \in \text { Strats }: \forall \text { sys } \in \text { Systs }: \text { sys conforms_to spec } \Rightarrow \\
\exists m \in \mathbb{N}: \text { poss_outs }\left(\text { sys }, \exp _{m}\right) \subseteq \text { validating }\left(\text { spec }, \exp _{m}\right) .
\end{gathered}
$$

In Example A, spec ${ }_{A 1}$ is strategically strongly D-validatable but not strongly D-validatable.

Other examples teach us that strategic strong D-refutability and strategic strong D-validatability are independent and not always fulfilled. In Example B,

- spec $_{B 1}$ is strategically strongly D-refutable but not strategically strongly $\mathrm{D}$-validatable, because the first figure 1 will show non-conformance, while no observation even of terribly many 0 's will ever tell whether the next sequence member is also a 0 ,

- $\operatorname{spec}_{B 2}$ is strategically strongly D-validatable but not strategically strongly D-refutable, because the first figure 1 will show conformance, while the absence of any 1 in the figures to come will never be established,

- $\operatorname{spec}_{B 3}$ is neither strategically strongly D-validatable nor strategically strongly D-refutable; in fact, $\operatorname{spec}_{B 3}$ is void, cf. [2].

\subsection{VAIN EXTENSIONS OF THE TAXONOMY OF TESTABILITY}

The testability notions defined in this section turn out to be equivalent to testability notions listed in Section 3.2. However, they help us to prove useful theorems about the true extensions defined in the previous section.

In OrdExpCont, a non-void specification spec is strategically weakly Drefutable if, with an appropriate sequence of experiments, some given nonconforming system could definitely be shown to be non-conforming, i.e. if

$$
\begin{aligned}
& \exists\left(\exp _{n}\right)_{n=1,2, \ldots} \in \text { Strats }, \text { sys } \in \text { Systs }, m \in \mathbb{N}: \\
& \text { poss_outs }\left(\text { sys }, \exp _{m}\right) \subseteq \text { invalid }\left(\text { spec }, \exp _{m}\right) .
\end{aligned}
$$

Lemma 1 Strategic weak D-refutability is equivalent to weak D-refutability.

Proof of Lemma $1 \Rightarrow$ : Let sys $\in$ Systs be a system and $\exp _{m}$ an experiment in a strategy $\left(\exp _{n}\right)_{n=1,2, \ldots}$ such that poss_outs $\left(\right.$ sys, $\left.\exp _{m}\right) \subseteq \operatorname{invalid}\left(\right.$ spec, $\left.\exp _{m}\right)$. 
Take $\exp :=e x p_{m}$, then the definition of weak D-refutability is fulfilled. $\Leftarrow$ : Let sys $\in$ Systs be a system and exp $\in$ Exps an experiment such that poss_outs $($ sys, $\exp ) \subseteq$ invalid (spec, exp $)$. Take $\forall n \in \mathbb{N}: \exp _{n}:=\exp$ as a strategy $\left(\exp _{n}\right)_{n=1,2, \ldots}$, then the definition of strategic weak D-refutability is fulfilled.

In OrdExpCont, a non-contradictory specification spec is strategically weakly $D$-validatable if, with an appropriate sequence of experiments, some given conforming system could definitely be shown to be conforming, i.e. if

$$
\begin{aligned}
& \exists\left(\exp _{n}\right)_{n=1,2, \ldots} \in \text { Strats }, \text { sys } \in \text { Systs }, m \in \mathbb{N}: \\
& \quad \text { poss_outs }\left(\text { sys }, \exp _{m}\right) \subseteq \text { validating }\left(\text { spec }, \exp _{m}\right) .
\end{aligned}
$$

Lemma 2 Strategic weak D-validatability is equivalent to weak D-validatability.

Proof of Lemma $2 \ldots$ analogous to that of Lemma 1.

Strategic weak D-refutability and strategic weak D-validatability do not characterize anything new as they are equivalent to weak D-refutability and weak D-validatability respectively (Lemma 1 and Lemma 2). In other words, if it comes to the certainty of refuting or validating only some systems, then unbounded strategies do not permit more insight than well-chosen simple experiments.

We encounter similar relationships if we attempt to carry over the notions of ordinary "experimental" ND-testability to strategic ND-testability. Strategic ND-refutability amounts to ND-refutability; strategic weak ND-validatability amounts to weak ND-validatability. As we do not need these two notions for proving the theorems about the true extensions of the taxonomy of testability, we omit the full definitions and lemmas for these notions and restrict ourselves to strategic strong ND-validatability.

In OrdExpCont, a non-contradictory specification spec is strategically strongly $N D$-validatable if, with an appropriate sequence of experiments, any given conforming system could be shown to be conforming, though possibly only with luck, i.e. if

$$
\begin{aligned}
& \exists\left(\exp _{n}\right)_{n=1,2, \ldots} \in \text { Strats }: \forall \text { sys } \in \text { Systs }: \text { sys conforms_to spec } \Rightarrow \\
& \quad \exists m \in \mathbb{N}, \text { out } \in \text { validating }\left(\text { spec }, \exp _{m}\right):\left(\text { sys }, \exp _{m}\right) \text { may_yield out. }
\end{aligned}
$$

Lemma 3 Strategic strong ND-validatability is equivalent to strong ND-validatability.

Proof of Lemma $3 \ldots$ analogous to that of Lemma 1. 


\subsection{EXTENDED TAXONOMY OF TESTABILITY}

Theorem 1 Strong D-refutability implies strategic strong D-refutability.

Proof of Theorem 1 Let $\exp \in$ Exps be an experiment such that

$\forall$ sys $\in$ Systs $: \neg($ sys conforms_to spec $) \Rightarrow$

poss_outs (sys, exp) $\subseteq$ invalid (spec, exp).

Take $\forall n \in \mathbb{N}: \exp _{n}:=\exp$ as a strategy $\left(\exp _{n}\right)_{n=1,2, \ldots}$, then the definition of strategic strong D-refutability is fulfilled.

Theorem 2 Strong D-validatability implies strategic strong D-validatability.

Proof of Theorem $2 \ldots$ analogous to the proof of Theorem 1.

Theorem 3 Strategic strong D-refutability implies weak D-refutability.

Proof of Theorem 3 Strategic strong D-refutability obviously implies strategic weak D-refutability, which is equivalent to weak D-refutability (Lemma 1).

Theorem 4 Strategic strong D-validatability implies weak D-validatability.

Proof of Theorem 4 Strategic strong D-validatability obviously implies strategic weak D-validatability, which is equivalent to weak D-validatability (Lemma 2).

Theorem 5 Strategic strong D-validatability implies strong ND-validatability.

Proof of Theorem 5 Strategic strong D-validatability obviously implies strategic strong ND-validatability, which is equivalent to strong ND-validatability (Lemma 3).

Summarizing the implications between experimental and strategic testability properties, we can extend the taxonomy of testability as depicted in Figure 3.

\section{CONCLUSION AND OUTLOOK}

As the history of testability notions shows, testability has even more flavours than those offered in the present and its companion paper [3]. It seems to be advisable to define clearly the various testability notions and to distinguish carefully among them. Otherwise, general remarks about testability are prone to ambiguity or meaningless. 


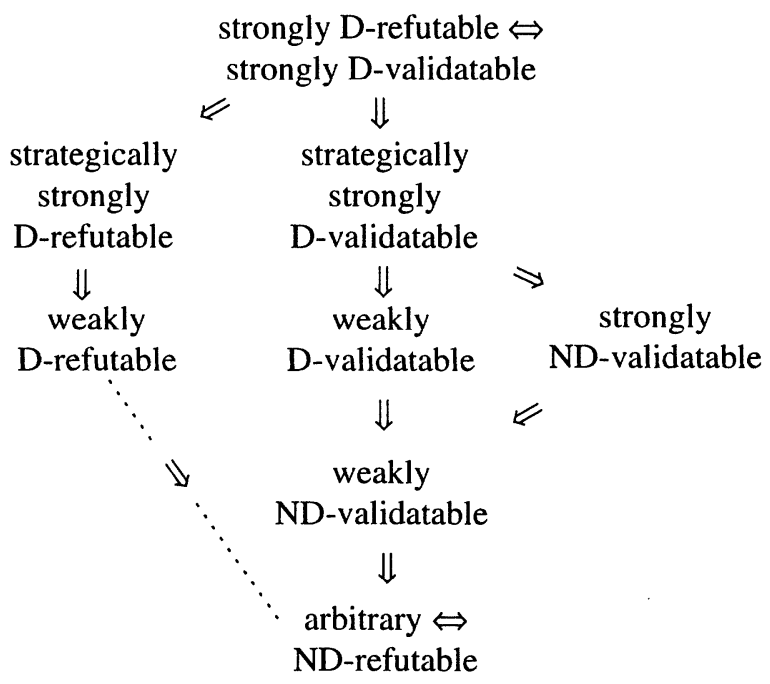

Figure 3 Implications between experimental and strategic testability properties.

As in most practical specification contexts systems may exhibit non-deterministic and infinite behaviour and validating outcomes do not exist, the practically most relevant testability property is ND-refutability. This is the weakest testability property and is held by any non-void specification.

It is worth future efforts to dedicate the testability notions for practical specification contexts and to quantify them.

\section{Acknowledgments}

We gratefully acknowledge the very helpful discussions with Helmut Wiland.

\section{References}

[1] B. Baumgarten. Die algebraische Spezifikation von Prozessen. Course notes, WS 1992/93, TH Darmstadt.

[2] B. Baumgarten. The Observational Significance of System Requirements. In preparation, cf. [4].

[3] B. Baumgarten and H. Wiland. Qualitative notions of testability. In: A. Petrenko and N. Yevtushenko (eds.), Testing of Communicating Systems, pp. 349-364, 1998, Kluwer Academic Publishers.

[4] B. Baumgarten, O. Henniger, and H. Wiland. Web pages on specification theory in the ASPEKTE project. 1999, http: //www.darmstadt.gmd.de/ baumgart/aspekte.html. 
[5] B. Beizer. Software Testing Techniques, Second edition. 1990, Van Nostrand Reinhold.

[6] E. Brinksma et al. A Formal Approach to Conformance Testing. In: J. de Meer, L. Mackert, W. Effelsberg (eds.), Protocol Test Systems, pp. 349-363, 1990, Elsevier/North-Holland.

[7] R. Nahm. Conformance testing based on formal description techniques and message sequence charts. $\mathrm{PhD}$ thesis, 1994, University of Bern, Switzerland.

[8] A. Petrenko, R. Dssouli, and H. König. On Evaluation of Testability of Protocol Structures. In: O. Rafiq (ed.), Protocol Test Systems VI, pp. 111-123, 1994, Chapman\&Hall.

[9] J.M. Voas and K.W. Miller. Software Testability: The New Verification. IEEE Software, May 1995.

[10] S.T. Vuong, A.A.F. Loureiro, S.T. Chanson. A Framework for the Design for Testability of Communication Protocols. In: O. Rafiq (ed.), Protocol Test Systems VI, pp. 89-108, 1994, Chapman\&Hall. 